\title{
Nuevo infiltrómetro inundador para test de infiltración económico en terrenos agroforestales y espacios verdes
}

\author{
Mongil, J. ${ }^{*}$, Navarro, J. ${ }^{2}$, Díaz V. ${ }^{1}$, Cruz, V. ${ }^{1}$ \\ ${ }^{1}$ Grupo de Hidrología y Conservación de Aguas y Suelos. Universidad Católica de Ávila. \\ Canteros, s/n 05005-Ávila \\ ${ }^{2}$ Departamento de Ingeniería Agrícola y Forestal. Universidad de Valladolid. Av. Madrid, 57 34004-Palencia \\ *e-mail: jorge.mongil@ucavila.es
}

\section{Resumen}

En este trabajo se presenta un prototipo de infiltrómetro inundador mejorado de anillo simple (Infiltest). Comprende una serie de elementos que facilitan la realización de mediciones en campo. Además, el gasto de agua es reducido y el coste del propio aparato económico. Consta de un cilindro de metacrilato, sujeto mediante un cilindro de acero que se introduce varios centímetros en el terreno. El cilindro de metacrilato lleva una escala milimetrada para realizar las lecturas. Así mismo, una rejilla sujeta por unas pinzas regulables impide que se levanten restos vegetales durante el ensayo y evita la degradación de la superficie edáfica por el impacto del agua al rellenarse el cilindro.

El infiltrómetro está diseñado para realizar mediciones de infiltración en terrenos forestales, agrícolas, pastizales, jardines y campos deportivos de césped (fútbol, golf, etc.). Esto hace posible un mejor conocimiento de la infiltración con fines científicos, técnicos y pedagógicos, así como el mejor diseño de los equipos de riego, de las técnicas o medios de drenaje, transformaciones de secano a regadío y la valoración de la degradación de los suelos de uso ganadero o agroforestal. Igualmente permite caracterizar de forma económica la capacidad de los suelos para generar escorrentía y, por ello, facilita la predicción de los modelos hidrológicos para la estimación de los recursos hídricos, las crecidas e inundaciones y, por tanto, para el manejo del agua y la planificación del territorio.

Palabras clave: escorrentía, Infiltest, infiltrómetro de anillo simple, permeabilidad, tasa de infiltración, velocidad de infiltración. 


\section{Introducción}

La infiltración es uno de los principales componentes del ciclo hidrológico y, en los ecosistemas terrestres dependientes del agua, un verdadero detonante de relaciones ecológicas imprescindibles para su funcionamiento (Martínez de Azagra et al., 2006a; Martínez de Azagra et al., 2006b). Consiste en la entrada de agua en el suelo a través de su superficie, producido por fuerzas gravitacionales y capilares (Hewlett, 1982; Martínez de Azagra y Navarro, 1996). Se trata de un proceso muy estudiado y con numerosos intentos de modelización (Green and Ampt, 1911; Horton, 1937; Holtan, 1961; Overton, 1964; Martínez de Azagra et al., 2006a; etc.). Se conoce como intensidad, velocidad o tasa de infiltración a la cantidad de agua que pasa a través de la superficie del suelo en un determinado instante $\left(\mathrm{mm} \mathrm{h}^{-1}\right)$. Igualmente, la capacidad de infiltración es la cantidad máxima de agua que puede introducirse en un suelo por unidad de tiempo y en un instante dado.

La infiltración es un proceso complejo que depende de un buen número de factores. De forma sintética, estos factores son (Skaggs and Khaheel, 1982; Cerdà, 1995; Martínez de Azagra y Navarro, 1996): la precipitación (intensidad, duración, tamaño de las gotas); contenido previo de humedad en el suelo; las características del agua que se infiltra (turbidez, contenido en sales, temperatura, carga de agua sobre el suelo); topografía (pendiente y exposición); vegetación (cubierta de plantas superiores y de musgos y líquenes); suelo (estado de la superficie del suelo, profundidad, pedregosidad, textura, estructura, densidad y porosidad, permeabilidad, materia orgánica, carbonato cálcico, estabilidad de agregados); y tratamientos del suelo (tratamientos agrosilvopastorales, pavimentación, selladores de suelos, compactación-descompactación). Al depender de tantos factores, el estudio y la modelización de la infiltración se complica de forma considerable. A esto hay que añadir la dificultad para obtener medidas precisas de esta variable. Por estos motivos, el conocimiento de este componente del ciclo hidrológico es insuficiente, especialmente en terrenos forestales, en los que se han llevado a cabo numerosas experiencias (Valeron and Meixner, 2010; Bonell et al., 2010; Alaoui et al., 2011; Mongil y Navarro, 2012) que subrayan la elevada variabilidad que se da en el proceso de infiltración.

Aunque existen diversas técnicas de cuantificación de la infiltración (Brakensiek et al., 1979; Hillel, 1980; Haan et al., 1982; Cerdà, 1995), uno de los problemas en el estudio de este fenómeno es la dependencia del método de medición. Las dos técnicas más utilizadas y aceptadas son los infiltrómetros inundadores y los simuladores de lluvia (Sidiras and Roth, 1987; Cerdà, 1995; Aoki and Sereno, 2006). No obstante, algunos estudios han obtenido con infiltrómetro inundador valores de la tasa de infiltración unas ocho veces mayores que con simulación de lluvia (Cerdà, 1995). Las intensidades de lluvia de la mayoría de los simuladores reproducen aguaceros de periodo de retorno elevados $\left(>50 \mathrm{~mm} \mathrm{~h}^{-1}\right)$ que en periodos de ensayo cortos no reflejan el comportamiento normal del suelo. Los infiltrómetros inundadores, en cambio, aunque se basan en la administración de una altura de agua determinada, aplicada con una carga hidrostática, no tienen ese efecto tan acusado de compac- 
tación del suelo y destrucción de la porosidad superficial, debido a la energía cinética de las gotas de los simuladores.

Existen en el mercado numerosos tipos y modelos de infiltrómetros y otros empleados también por diferentes autores en sus ensayos (Tricker, 1978; Blanco, 1999; Gazol et al., 2002; Simoes et al., 2005; Xua et al., 2012). Los infiltrómetros inundadores más sencillos consisten en un cilindro hueco (infiltrómetro de anillo simple) o dos cilindros concéntricos (infiltrómetro de doble anillo). El material empleado suele ser acero, aunque también existen infiltrómetros de materiales plásticos. La utilización de materiales opacos dificulta la medición del nivel de agua. Respecto al tamaño de los cilindros, oscila entre $2 \mathrm{~cm}$ y $1.2 \mathrm{~m}$ de diámetro aproximadamente, según los diferentes autores. Por otra parte, los infiltrómetros inundadores pueden ser de carga constante, si tienen siempre la misma altura de agua, o de altura variable, cuando sólo se rellenan cuando baja sensiblemente la lámina de agua. Es importante destacar que los infiltrómetros más sofisticados y/o de mayor tamaño tienen un complejo uso en campo, lo que dificulta las mediciones especialmente en terrenos abruptos e inaccesibles. A mayor diámetro de cilindro resulta necesaria una mayor cantidad de agua para realizar los ensayos, lo que supone inconvenientes para su transporte, especialmente en terrenos de difícil acceso. También resulta más difícil su instalación en terrenos heterogéneos, pues es más fácil interceptar piedras, rocas o grandes raíces. Además de los ya indicados, otros inconvenientes frecuentes en la utilización de infiltrómetros son: dificultad para introducir el cilindro en el suelo cuando se utilizan cilindros de pared gruesa; tropiezo de las escalas del flotador con elementos del suelo difíciles de detectar durante la medición (piedras, hinchado del suelo, hojarasca...) con infiltrómetros opacos; errores en la medición al utilizar cilindros de pequeño tamaño (Blanco, 1999); y compactación del suelo y levantamiento de materiales sólidos de su superficie, como hojarasca, pinocha, otros restos vegetales, limos y arcillas, etc.

\section{Objetivos}

El objetivo de este trabajo es presentar un nuevo infiltrómetro inundador que, con una serie de mejoras que facilitan la medición, permite realizar ensayos de infiltración en campo de forma sencilla y económica.

\section{Descripción detallada del infiltrómetro}

El infiltrómetro que se ha diseñado en este trabajo (solicitud de patente P201331510) es un infiltrómetro inundador de anillo simple y carga variable. Este tipo de infiltrómetro ya existe en el mercado, pero se ha introducido un protocolo de campo y una serie de elementos que mejoran las mediciones y palian buena parte de los inconvenientes que suelen surgir cuando se utilizan infiltrómetros de anillo simple y de diámetro pequeño, que fundamentalmente son filtraciones late- 
rales y menor precisión en la medida. El infiltrómetro diseñado (Infiltest) es sencillo, económico, con bajo consumo de agua y consta de los siguientes elementos (Fig. 1 y 2 y Tab. 1): un cilindro de acero galvanizado, para facilitar la inserción y
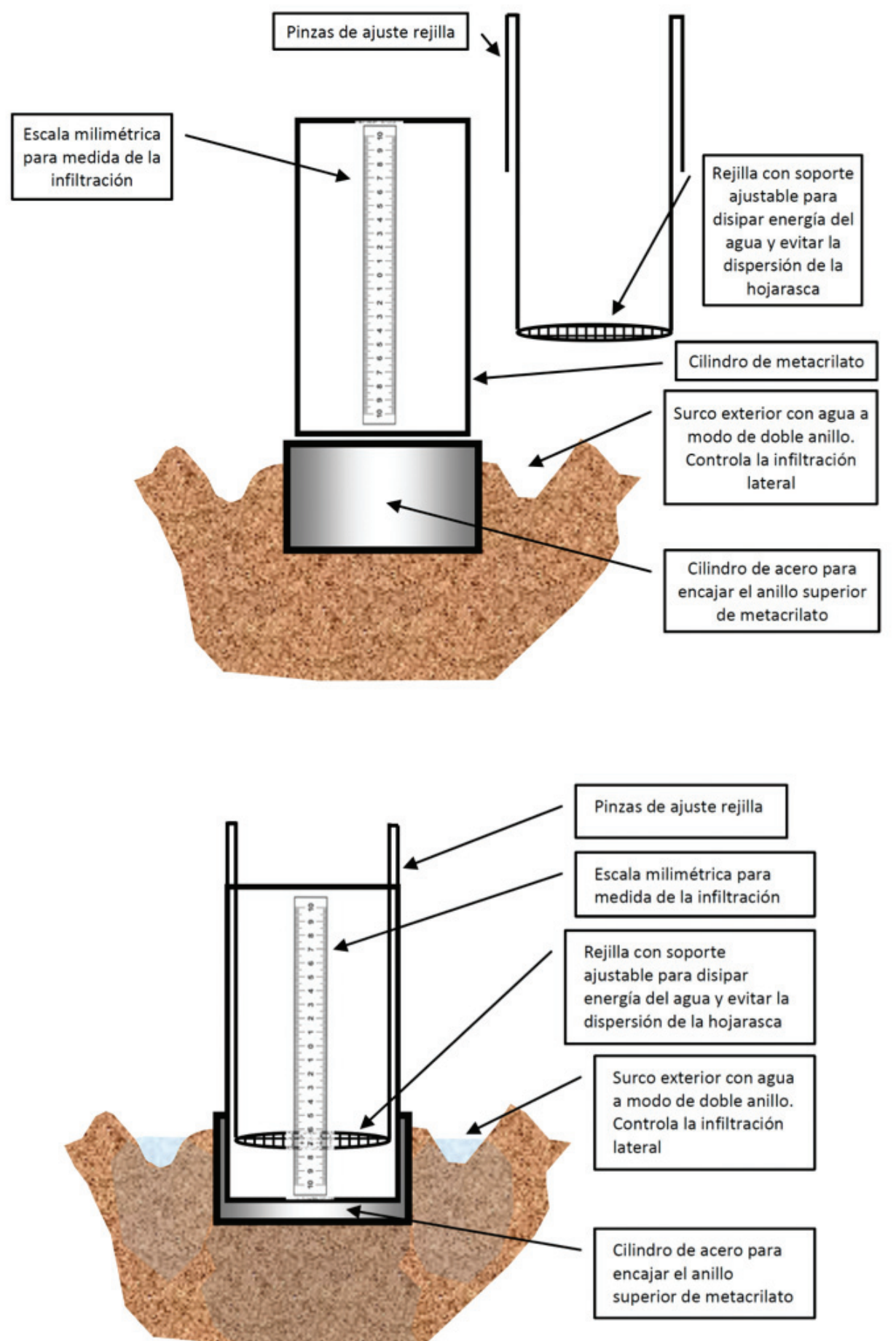

Figura 1. Elementos que componen Infiltest. 


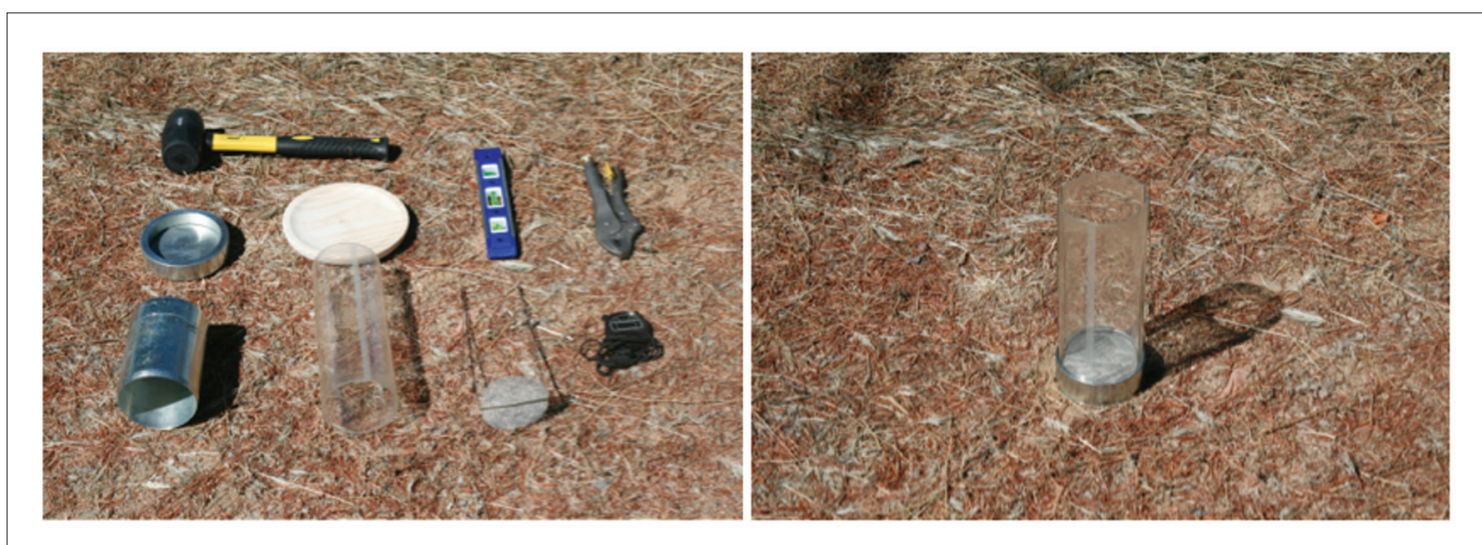

Figura 2. Infiltest desmontado con todos sus componentes e instalado en campo.

sujeción en el suelo de un cilindro de metacrilato, que a su vez posee una escala de medición, y una rejilla con pinzas de fijación a altura variable. El equipo de medición se completa con: una plancha de acero y una tabla de madera que facilitan el clavado en el suelo del cilindro de acero, una maza de goma, un nivel de burbuja, una mordaza, un cronómetro, unos bidones de plástico con embudo y los estadillos de campo.

Las dimensiones de los cilindros de acero y metacrilato y de la rejilla se muestran en la Tab. 1. El cilindro de acero lleva grabada una señal que lo divide en dos tramos, uno de $10 \mathrm{~cm}$ (que se introduce en el suelo) y otro de $3 \mathrm{~cm}$ (que queda en el exterior). El ajuste del cilindro de acero con el de metacrilato se realiza mediante cinta de fontanero. El cilindro de metacrilato lleva una regla milimetrada grabada con láser, para realizar las mediciones. El metacrilato, frente al acero, es a la vez transparente y resistente, por lo que permite conocer posibles incidencias ocurridas durante el experimento y medir la altura de agua con mayor precisión. Las dimensiones de este cilindro único hacen que tenga un menor gasto de agua (se estima que el consumo de agua es, respectivamente para suelo arcilloso y arenoso, entre 2 y 14 veces menor que con un infiltrómetro de cilindro simple de $30 \mathrm{~cm}$ ), y sea más cómodo de utilizar en terrenos en pendiente. Las filtraciones laterales se controlan mediante la excavación de un surco de unos $5-10 \mathrm{~cm}$ de profundidad alrededor del cilindro, que se llena de agua cada cierto tiempo, como se explicará más adelante. La pequeña altura implica una reducción del error, ya que, según Blanco (1999), una carga de agua de entre 0 y $30 \mathrm{~cm}$ no hace variar excesivamente la infiltración. La rejilla es de aluminio y de forma circular; va sujeta al cilindro de metacrilato mediante unas pinzas realizadas con flejes de acero. Su función es evitar la compactación del suelo y proteger su estructura natural al depositar el agua en el infiltrómetro, así como evitar que se levanten y queden en suspensión en el agua durante el ensayo materiales flotantes como restos vegetales preexistentes en la superficie del suelo. Las pinzas hacen posible la regulación de la altura de la rejilla dentro del cilindro, lo cual es especialmente importante en suelos arcillosos, donde el agua provoca su hinchamiento, o suelos con importante espesor de hojarasca. 
Tabla 1. Dimensiones de los elementos que componen Infiltest.

\begin{tabular}{|c|c|}
\hline ELEMENTO & DIMENSIONES \\
\hline \multirow{3}{*}{ Cilindro de acero } & Altura: $13.0 \mathrm{~cm}$ \\
& Diámetro interior: $9.0 \mathrm{~cm}$ \\
& Diámetro exterior: $9.6 \mathrm{~cm}$ \\
& Grosor de pared: $0.3 \mathrm{~cm}$ \\
\hline \multirow{2}{*}{ Cilindro de metacrilato } & Altura: $24.8 \mathrm{~cm}$ \\
& Diámetro interior: $8.3 \mathrm{~cm}$ \\
& Diámetro exterior: $9.0 \mathrm{~cm}$ \\
\hline \multirow{2}{*}{ Rejilla con pinzas de sujeción de altura variable } & Grosor de pared: $0.35 \mathrm{~cm}$ \\
& Diámetro de la rejilla: $8.3 \mathrm{~cm}$ \\
& Luz: 0.5 mm \\
\hline
\end{tabular}

\section{Dónde utilizar el infiltrómetro}

Infiltest está diseñado para la medición de la infiltración de suelos en campo. Así, con un mínimo gasto de agua, puede utilizarse para estimar la permeabilidad en terrenos forestales, pastizales, terrenos agrícolas, jardines y campos deportivos de tierra o césped (fútbol, golf, etc.). Esto hace posible un mejor conocimiento de la infiltración con fines científicos, técnicos y pedagógicos, así como el mejor diseño de los equipos de riego y de las técnicas de drenaje.

Los estudios de infiltración son de gran interés en las transformaciones de secano a regadío y en la valoración de la degradación de los suelos de uso ganadero. Así mismo, el infiltrómetro permite caracterizar de forma económica la capacidad de los suelos para generar escorrentía y, por ello, una mejor predicción de los modelos hidrológicos para la estimación de crecidas e inundaciones $\mathrm{y}$, por tanto, para la planificación del territorio.

\section{Cómo utilizar el infiltrómetro}

El procedimiento de utilización del infiltrómetro tiene una primera parte de instalación y otra de realización del ensayo de infiltración.

\subsection{Instalación}

La instalación se realiza de la siguiente manera:

1. Se busca una ubicación adecuada para el ensayo, con características edáficas medias dentro del entorno a analizar. Debe elegirse una ubicación sin afloramientos rocosos ni grandes piedras que puedan dañar al infiltrómetro en su instalación. 
2. Se clava el cilindro de acero, hasta una profundidad recomendada de $10 \mathrm{~cm}$ y mínima de $5 \mathrm{~cm}$. Especialmente en suelos arcillosos, con grietas o con abundante materia orgánica, la profundidad de inserción debe ser mayor de 5 $\mathrm{cm}$. Para el clavado del cilindro, éste se sitúa en el suelo y se coloca sobre él la plancha de acero. Golpeando cuidadosamente sobre la plancha se va introduciendo el cilindro, intentando no alterar la estructura del suelo. Mediante el nivel de burbuja se comprueba que el cilindro está situado en posición perfectamente vertical.

3. Se introduce el cilindro de metacrilato dentro del cilindro de acero, de manera que quede ajustado de forma adecuada.

4. Se coloca la rejilla en su posición, quedando sujeta al cilindro de metacrilato mediante las pinzas. La regulación de la altura de la rejilla sobre el suelo dependerá fundamentalmente del contenido en arcillas expansivas que tenga el suelo. Se recomienda situarla al menos a $5 \mathrm{~cm}$ del suelo.

5. Alrededor del infiltrómetro, separada de este unos $2 \mathrm{~cm}$ para evitar que se descalce, se cava una pequeña zanja circular de $5-10 \mathrm{~cm}$ de profundidad y lo mismo de anchura, que se rellena de agua cada cierto tiempo durante el ensayo, para controlar la infiltración lateral.

6. Una vez terminado el ensayo, el desmontaje del infiltrómetro se realiza con el procedimiento inverso, teniendo en cuenta que el cilindro de acero puede extraerse utilizando la mordaza.

\subsection{Ensayo de infiltración}

El protocolo del ensayo de infiltración es el siguiente:

1. Se rellena el estadillo, en el que se anotan condiciones ambientales, como la temperatura, y características de la superficie del suelo.

2. Se realizan como mínimo tres replicaciones en cada suelo en una parcela circular de $10 \mathrm{~m}$ de diámetro para disminuir la variabilidad del suelo.

3. El infiltrómetro se llena de agua hasta el borde superior (encharcamiento o inundación). La altura del agua dependerá de la profundidad a la que se haya clavado el infiltrómetro, pero estará entre 15 y $22 \mathrm{~cm}$. Se mide la altura de agua infiltrada en unos intervalos de tiempo dados (cada 2 minutos al principio del ensayo y cada 30 minutos al final). Después de cada medición se rellena el cilindro inmediatamente y se humedece la zona colindante, como aconseja, por ejemplo, Harvey (1982).

4. El ensayo se prolonga hasta que la tasa de infiltración se estabilice en torno a un valor, lo que suele producirse aproximadamente a las 3 ó $4 \mathrm{~h}$ del inicio del ensayo.

\section{Agradecimientos}

Este proyecto se ha desarrollado gracias a la financiación de T-CUE4, dentro de 
las ayudas para el desarrollo de proyectos del programa piloto de prototipos orientados al mercado, y no hubiera sido posible sin el apoyo técnico del personal de la Oficina de Transferencia de Resultados de la Investigación (OTRI) de la Universidad Católica de Ávila. También ha sido imprescindible la colaboración de Maquinaria Agrícola Cruz, S.L., en especial de José Carlos Cruz.

\section{Bibliografía}

Alaoui, A., Caduff, U., Gerke, H.H., Weingartner, R., 2011. Preferential flow effects on infiltration and runoff in grassland and forest soils. Vadose Zone J. 10(1), 367-377. doi: $10.2136 /$ vzj2010.0076

Aoki, A.M., Sereno, R., 2006. Evaluación de la infiltración como indicador de calidad de suelo mediante un microsimulador de lluvias. Agriscientia XXIII (1), 23-31.

Blanco, R., 1999. El infiltrómetro de cilindro simple como método de cálculo de la conductividad hidráulica de los suelos. Experiencias de campo en ámbitos de montaña mediterránea. Baetica. Estudios de Arte, Geografía e Historia 21, 9-33.

Bonell, M., Purandara, B.K., Venkatesh, B., Krishnaswamy, J., Acharya, H.A.K., Singh, U.V., Jayakumar, R., Chappell, N., 2010. The impact of forest use and reforestation on soil hydraulic conductivity in the Western Ghats of India: implications for surface and subsurface hydrology. J. Hydrol. 391(1-2), 49-64. doi:10.1016/j.jhydrol.2010.07.004

Brakensiek, D.L., Rawls, W.J., Hamon, W.R., 1979. Aplication of an infiltrometer system for describing infiltration into soils. Transactions of the ASAE 22, 320-333.

Cerdà A., 1995. Factores y variaciones espacio-temporales de la infiltración en los ecosistemas mediterráneos. Monografías científicas $n^{\circ} 5$. Geoforma ediciones. Logroño.

Gazol, R., Amézqueta, E., Aragüés, R., 2002. Desarrollo de un infiltrómetro automático y su aplicación en campo. Investigación agraria. Producción y protección vegetales 17(1), 131-142.

Green, W.H., Ampt, C.A., 1911. Studies on soil physics, I. Flow of air and water through soils. J. Agr. Sci. 4, 1-24.

Haan, C.J., Johnson, H.P., Brakensiek, D.L., 1982. Hydrologic modeling of small watersheds. ASAE monograph 5. American Society of Agricultural Engineers. St Joseph, MI.

Harvey, A.M., 1982. The role of piping in the development of badlands and gully systems in south-east Spain. In: Bryan, R., Yair, A. (Eds.), 1982. Badland geomorphology and piping. University Press. Cambridge.

Hewlett, J.D., 1982. Principles of forest Hydrology. The University of Georgia Press. Athens, GA. Hillel, D., 1980. Fundamentals of soil physics. Academic Press. London.

Holtan, H.N., 1961. A concept for infiltration estimates in watershed engineering. Agricultural Research Service Paper, 41-51. ARS, USDA. Washington D.C.

Horton, R.E., 1937. Determination of infiltration capacity for large drainage basins. Trans. Am. Geophys. Union 18, 371-385.

Martínez de Azagra, A., Navarro, J., 1996. Hidrología forestal. El ciclo hidrológico. Servicio de publicaciones de la Universidad de Valladolid. Valladolid.

Martínez de Azagra, A., Pando, V., Del Río, J., Navarro, J., 2006a. Aproximación al conocimiento de la infiltración a través del análisis dimensional. Ecología 20, 471-491. 
Martínez de Azagra, A., Pando, V., Navarro, J., Del Río, J., 2006b. A proposal of an infiltration function with ecological meaning. International meeting IUFRO. Managing Forest Ecosystems: The Challenges of Climate Change. Procedings. Palencia.

Mongil, J., Navarro, J., 2012. Infiltración y grupos hidrológicos de suelos en las laderas de los páramos (Valladolid). Cuadernos de Investigaciones Geográficas 38, 131-153.

Overton, D.E., 1964. Mathematical refinement of an infiltration equation for watershed engineering. USDA-Agricultural Research Service. Washington D.C.

Sidiras, N., Roth, C.H., 1987. Infiltration measurements with double-ring infiltrometers and a rainfall simulator under different surface conditions on an oxisol. Soil Tillage Res. 9 (2), 161-168.

Simoes, W.L., Figueiredo, V.B., Da Silva, E.L., 2005. Uso do cilindro infiltrómetro único em diferentes solos. Eng. Agríc. Jaboticabal 25(2), 359-366.

Skaggs, R.W., Khaheel, R., 1982. Infiltration. In: Haan, C.T., Johnson, H.P., Brakensiek, D.L., (Eds.), 1982. Hydrologic modeling of small watersheds. ASAE Monograph 5. St. Joseph, MI.

Tricker, A.S., 1978. The infiltration cylinder: some comments on its use. J. Hydrol. 36, 383391.

Valeron, B., Meixner, T., 2010. Overland flow generation in chaparral ecosystems: temporal and spatial variability. Hydrol. Process. 24 (1), 65-75. DOI: 10.1002/hyp.7455.

Xua, X., Lewis, C., Liu, W., Albertson, J.D., Kiely, G., 2012. Analysis of single-ring infiltrometer data for soil hydraulic properties estimation: comparison of BEST and Wu methods. Agr. Water Manage. 107, 34-41. doi:10.1016/j.agwat.2012.01.004. 
